\title{
Anabases
}

ANABASES Traditions et réceptions de l'Antiquité

$10 \mid 2009$

Varia

\section{Kurt A. RAAFlaub (éd.), War and Peace in the Ancient World}

\section{Geneviève Hoffmann}

\section{OpenEdition}

\section{Journals}

Édition électronique

URL : http://journals.openedition.org/anabases/737

DOI : 10.4000/anabases.737

ISSN : 2256-9421

\section{Éditeur}

E.R.A.S.M.E.

\section{Édition imprimée}

Date de publication : 1 octobre 2009

Pagination : 299-302

ISSN : 1774-4296

\section{Référence électronique}

Geneviève Hoffmann, «Kurt A. raaflaub (éd.), War and Peace in the Ancient World ", Anabases [En ligne], 10 | 2009, mis en ligne le 01 juillet 2011, consulté le 22 septembre 2020. URL : http:// journals.openedition.org/anabases/737 ; DOI : https://doi.org/10.4000/anabases.737

Ce document a été généré automatiquement le 22 septembre 2020.

(c) Anabases 


\title{
Kurt A. RAAFlaub (éd.), War and Peace in the Ancient World
}

\author{
Geneviève Hoffmann
}

\section{RÉFÉRENCE}

Kurt A. RAAFLAUB (éd.), War and Peace in the Ancient World, Malden, Blackwell Publishing Ltd, 2007, $385 \mathrm{p}$.

39,95 dollars / ISBN 978-1-4051-45266-8.

1 Avec ce volume sur la guerre et la paix dans les mondes anciens, Kurt A. Raaflaub (KAR) ouvre la collection d'études comparées qu'il dirige, la notion d'ancienneté étant à comprendre au sens large dans un espace qui ne l'est pas moins, puisque dans le cas présent, le lecteur est conduit au fil des dix-neuf contributions, de la chine à l'Amérique, en passant par les mondes méditerranéens. Le propos de la collection est d'exposer la diversité des réponses apportées à des défis semblables par des modèles sociaux différents.

2 Ce sont les actes du colloque sur « la guerre, la paix et la réconciliation » (2002-2003, Brown University), qui sont publiés dans le présent ouvrage. Dans l'introduction (p. 1-33), précédée par la présentation des auteurs, KAR formule d'emblée l'axe majeur du projet : comment déceler, au-delà des sources, au-delà de la fureur des armes et de la gloire projetée par les conquérants, la place qu'occupe la paix dans les sociétés antiques, dans leur système religieux et leurs modes de pensée ? Cette question le conduit à présenter des pistes de recherche jalonnées de références bibliographiques qui permettent d'approcher l'historiographie du sujet sans toutefois l'épuiser. L'auteur clôt sa présentation sur la place exceptionnelle qu'occupent les Grecs dans cette réflexion, et qu'il justifie par des raisons historiques et littéraires.

3 L'ordre adopté pour la présentation des chapitres traduit deux impératifs, l'un géographique, l'autre chronologique. Si le cœur du volume est formé par les six contributions sur le monde gréco-romain, l'espace parcouru d'est en ouest conduit le 
lecteur de la civilisation chinoise, jugée la plus éloignée culturellement du modèle occidental, à la Ligue Iroquoise, étudiée jusqu'en 1777. Dans ces mondes dits " anciens » où la guerre est non seulement une donnée permanente mais aussi le fondement de l'organisation sociale, la paix offre différentes facettes que révèle l'étude du vocabulaire autant que celle des stratégies mises en œuvre pour en créer les conditions et en assurer la pérennité.

4 Robin D. S. Yates (chapitre 2, p. 34-52) propose une étude historique des manières de faire la guerre et la paix en Chine dans la période appelée des Printemps et Automnes et les Royaumes Combattants, c'est-à-dire entre 722 et 221 . Ces temps troublés furent aussi ceux de l'éclosion d'un des plus importants courants de pensée, celui de Confucius. S'est alors développée une réflexion sur les conditions requises pour maintenir l'harmonie du monde et pour tenter de définir «la guerre juste», la paix restant conçue comme une trêve. L'étude de Richard Salomon (chapitre 3, p. 53-65) prend comme point de départ l'image traditionnelle de l'Inde comme terre de nonviolence. L'analyse du vocabulaire de la paix en sanscrit dans une littérature qui ignore le genre historique (p. 53), conduit l'auteur à souligner que les Indiens n'ont jamais eu contrairement aux Grecs et aux Romains de déesse de la Paix et qu'à leurs yeux la paix ne peut être qu'intérieure: vouloir changer le monde dominé par des conflits incessants est une perte de temps. Ces deux premières communications exposent des thèmes que l'on retrouve dans les études qui suivent, qu'elles portent sur le vocabulaire, les pratiques diplomatiques, l'idéologie de la paix ou les images des sociétés transmises par l'historiographie, chaque thème étant plus ou moins développé en fonction des sources et de la perspective adoptée par l'auteur. Ainsi Benjamin R. Foster (chapitre 4, p. 66-80) s'emploie-t-il à mettre à mal l'image belliqueuse associée aux Mésopotamiens en raison des sources iconographiques et littéraires, car leur société a su développer des mécanismes pour établir la paix. Deux contributions ont trait aux Hittites. Celle de Richard H. Beal (chapitre 5, p. 81-97) relève que la paix est une construction fragile qui nécessite une activité diplomatique, le maillage du territoire par des garnisons, des alliances matrimoniales sans oublier le concours des dieux. Lanny Bell (chapitre 6, p. 98-120) s'intéresse au premier grand traité de paix entre deux superpuissances, en l'occurrence l'Égypte et l'État hittite, conclu après la bataille de Qadesh, et à ses implications sur l'image que les Egyptiens ont eue des étrangers. Sur plus de 1000 ans d'histoire de l'Iran, Josef Wiesehöfer (chapitre 7, p. 121-140) remet en question la réputation faite par l'historiographie aux Achéménides, aux Parthes et aux Sassanides en soulignant combien les Parthes, injustement traités par la tradition et la recherche, méritent d'être réhabilités pour avoir sur préserver la paix dans leur empire. Susan Niditch (chapitre 8, p.141-160) et Thomas Krüger (chapitre 9, p.161-171) traitent de la paix et de la réconciliation dans le royaume d'Israël, la première d'un point de vue historique, le second en se concentrant sur la prophétie du Livre de Michée (4, 1-5) qui annonce la fin de la guerre, grâce au triomphe de la Loi et de la Justice quand avec le concours de Dieu « de leurs glaives ils feront des hoyaux et de leurs lances des serpes ».

5 Six contributions portent sur le monde gréco-romain dont l'originalité prend sens au regard des autres cultures. Les deux premières exploitent les sources littéraires, d'Homère à Euripide. Lawrence A. Tritle (chapitre 10, p. 172-190) souligne que dès l' Iliade, apparaît une dénonciation des horreurs de la guerre et une célébration de la paix. David Konstan (chapitre 11, p. 191-205) centre son étude sur le thème de la vengeance et des possibilités de la réconciliation dans l'univers d'Euripide. Victor Alonso dresse 
une typologie des relations entre cités, des spondai jusqu'à l'apparition d'un droit international (chapitre 12, p. 206-225). La pax romana des $\mathrm{I}^{\mathrm{er}}$ et $\mathrm{II}^{\mathrm{e}}$ siècles ne doit pas masquer d'après Nathan Rosenstein (chapitre 13, p. 226-244) que pour Rome la paix n'a pas le même sens que pour nous, qu'elle signifie la capitulation et l'entrée dans la fides $\mathrm{du}$ vainqueur, donc un état précaire. Carlin A. Barton (chapitre 14, p. 245-255) s'intéresse au vocabulaire politique pour souligner que l'Ara Pacis signifie la fin des guerres civiles et donc la soumission des citoyens romains à l'autorité d'Auguste qui en a fait des sujets. L'analyse de Jeri Blair DeBrohun (chapitre 15, p. 256-278) porte sur les Portes de la Guerre (et de la Paix) comme motif littéraire dans les Res Gestae d'Auguste, et les œuvres de Tite-Live, de Virgile, d'Horace et d'Ovide.

6 Avec Louis J. Swift (chapitre 16, p. 279-296), on retrouve la Bible pour s'intéresser à l'originalité du message de paix délivré par les chrétiens et à sa signification, avant et après l'adoption du christianisme comme religion d'État par Constantin. Fred M. Donner (chapitre 17, p. 297-311) relève combien le Coran est ambivalent, puisque la lutte contre les incroyants que chaque bon musulman doit mener, est associée à la notion de compassion. Les trois dernières communications conduisent le lecteur chez les Aztèques (Ross Hassig, chapitre 18, p. 312-328), les Incas (Catherine Julien, chapitre 19, p. 329-347) et les Iroquois (Neta C. Crawford, chapitre 20, p. 348-368). À une société belliqueuse qui n'accorde aucune place à la paix si ce n'est sous forme de domination, dans la conception d'un monde hiérarchisé dont les Aztèques occupent le sommet, s'oppose l'image d'une pax Incaica, tandis que les Iroquois sont crédités d'avoir su préserver la paix par leur Ligue, regroupement de cinq Nations, dont la politique se rapprocherait de l'idéal kantien.

$7 \quad$ L'index permet de croiser les informations d'un recueil dont cette présentation ne peut épuiser la richesse. Il appartiendra au lecteur de prendre connaissance de chacune de ces contributions qui sous forme de dossiers dotés d'une bibliographie, présentent les sources exploitables, dégagent l'image de chacun de ces mondes anciens, et proposent des pistes de recherche pour développer une approche comparatiste que ce soit pour les stratégies diplomatiques, pour les définitions des états de paix ou pour les théories philosophiques. On peut regretter que la part de l'économie (les profits de guerre contre les avantages de la pacification) soit négligée au bénéfice des pratiques mises en œuvre pour faciliter la réconciliation et instaurer la paix. Dans certaines communications, des comparaisons sont proposées avec des situations contemporaines qui sans être maladroitement anachroniques, reflètent néanmoins des partis pris politiques contestables. De ces études il ressort que si la paix se décline sous des formes différentes, elle n'est pas seulement l'envers de la guerre. Elle traduit toujours un rapport de forces, un équilibre précaire, un mode de relation avec les nations étrangères. Dans ce faisceau d'information, le modèle gréco-romain gagne en pertinence comme objet d'étude ce qu'il perd en spécificité dans le miroir de l'altérité. 


\section{AUTEURS}

\section{GENEVIĖVE HOFFMANN}

Université de Picardie Jules Verne

genevieve.hoffmann@wanadoo.fr 